\title{
The Design of Contactors Based on the Niching Multiobjective Particle Swarm Optimization
}

\author{
Wenying Yang $\mathbb{D}$, Jiuwei Guo $\mathbb{D}$, Yang Liu $(\mathbb{D}$, and Guofu Zhai $\mathbb{D}$ \\ School of Electrical Engineering and Automation, Harbin Institute of Technology, Harbin, Heilongjiang Province 150001, China \\ Correspondence should be addressed to Wenying Yang; yangwy@hit.edu.cn
}

Received 18 March 2018; Revised 19 April 2018; Accepted 2 May 2018; Published 4 July 2018

Academic Editor: Zhile Yang

Copyright (C) 2018 Wenying Yang et al. This is an open access article distributed under the Creative Commons Attribution License, which permits unrestricted use, distribution, and reproduction in any medium, provided the original work is properly cited.

\begin{abstract}
Contactors are important components in circuits. To solve the multiobjective optimization problems (MOPs) of contactors, a niching multiobjective particle swarm optimization (NMOPSO) with the entropy weight ideal point theory is proposed in this paper. The new algorithm selecting and archiving the nondominated solutions based on the niching theory to ensure the diversity of the nondominated solutions. To avoid missing the extreme solutions of each objective during the multiobjective optimization process, extra particle swarms used to search the independent optimal solution of each objective are supplemented in this algorithm. In order to determine the best compromise solution, a method to select the compromise solution based on entropy weight ideal point theory is also proposed in this paper. Using the algorithm to optimize the characteristics of a typical direct-acting contactor, the results show that the proposed algorithm can obtain the best compromise solution in MOPs.
\end{abstract}

\section{Introduction}

Contactors are very important electrical equipment used to control the circuit. The performance of contactors directly influences the safety and stability of the circuit. In recent years, with the improvement of the demand for contactor performance, the optimization problem of contactors has become a hot issue. The optimization problem of contactors is a typical multiobjective optimization problem. The characteristics and power consumption are all the optimization objectives. In the past, due to the limitation of calculation efficiency of the contactors' characteristics, the optimization problem of the contactor was mostly based on the orthogonal experiment which is more emphasized on the single-objective optimization $[1,2]$. In recent years, with the development of approximation models, the calculation efficiency of the contactors' characteristics has been improved. It is possible to apply multiobjective optimization algorithm to the optimization problem of contactors [3-5].

In the early days, the multiobjective optimization algorithm is realized by the linear weighting, setting constraints, and other methods which can integrate the multiobjective into a single objective. These methods were complicated and easily subjected to subjective experience. In recent years, with the development of intelligent optimization algorithms, combining the intelligent optimization algorithms with the Pareto optimal solution to achieve the multiobjective optimization has got wide attention. In all these algorithms, multiobjective genetic optimization (GA) algorithms developed fastest. Some of these algorithms are based on nondominance sorting such as nondominated sorting genetic algorithm (NSGA), nondominated sorting genetic algorithm II (NSGA-II), and niched Pareto genetic algorithm (NPGA) [6-9]. And some others are based on decomposition, such as multiobjective evolutionary algorithm based on decomposition (MOEA/D) and MOEA/D-M2M [10, 11]. All these multiobjective GA algorithms can realize the multiobjective optimization. However, due to the limitations of the GA, these algorithms still have some disadvantages in optimizing efficiency and effectiveness. Particle swarm optimization (PSO) algorithm is more effective than GA in many cases because of its simple operation, fast convergence rate, and excellent 
searching ability; some researchers study the multiobjective particle swarm optimization (MOPSO) [12-14]. Parsopoulos and Vrahatis have solved the dual-objective optimization problem by using a two-particle swarm optimization algorithm [15]. Coello et al. have proposed MOPSO based on adaptive grid and introduced the concept of archive [16]. Brits et al. have proposed a NichePSO based on the niching theory borrowed from the idea of EA which has been widely used for solving MOP [17]. In [12], Qu et al. have proposed a distance-based locally informed particle swarm (LIPS) and eliminate the need of niching parameter of PSO. In [18], a recently developed multiobjective particle swarm optimizer (D2MOPSO) is proposed; a new archiving technique that facilitates attaining better diversity is used in this algorithm. In [19], a dynamic multiple-swarm PSO in which the number of swarms is adaptively adjusted throughout the search process via the proposed dynamic swarm strategy is proposed. Lin et al. have proposed a novel MOPSO algorithm using multiple search strategies (MMOPSO), where two search strategies are designed to update the velocity of each particle [20]. Most of these algorithms focus on the selection of the best and the archiving strategy to make the solution set more diverse; some similar algorithms can be found in [21-24]. Campos et al. proposed a bare bone particle swarm optimization with scale matrix adaptation (SMA-BBPSO) to avoid premature convergence problem [25]. In [26], an information sharing mechanism (ISM) is proposed to improve the performance of a particle. In [27], Qin et al. has proposed an improved PSO algorithm with an interswarm interactive learning strategy (IILPSO) by overcoming the drawbacks of the canonical PSO algorithm's learning strategy. These scholars care more about the convergence and the learning strategy of PSO that improve the MOPSO indirectly [28-30]. Most of the above algorithms are based on archiving and these algorithms have been used in many fields [31-34].

The above MOPSO focuses on the diversity not the limiting case of each objective, so these algorithms often miss the independent optimal solution due to the selection and archiving strategy. At the same time, the results obtained by the above MOPSO are mostly Pareto optimal sets. In engineering, a compromise solution needs to be selected from the set. The above algorithms do not propose the method to select the compromise solution. In view of the above problems, this paper proposes a NMOPSO that considers the independent optimal solutions and gives a method to determine the compromise solution based on the entropy weight ideal point theory.

Taking a typical high-power DC contactor as an example, this paper first establishes an approximate model of its static and dynamic characteristics based on radial basis function (RBF) network to ensure that the computational efficiency of the contactor characteristics meets the need of the NMOPSO. Then, according to the NMOPSO proposed in this paper, the compromise solution is obtained. The objectives obtained before and after the optimization are compared to prove the effect of the algorithm. This multiobjective optimization algorithm is also suitable for the field of similar optimization problem.

\section{Approximate Model of Contactors' Static and Dynamic Characteristics}

The optimization objectives of contactors contain static and dynamic characteristics, power consumption, quality, and so on, so the optimization problem of contactors is a typical multiobjective optimization problem. Among all these optimization objectives, static and dynamic characteristics are the most important. They directly influence the contactors' switch speed and indirectly influence the life and reliability of contactors. The static characteristics of the contactors contain the electromagnetic flux and the electromagnetic force. The dynamic characteristics of the contactors include making time and speed of the armature. The dynamic characteristics can be solved by the 4th Runge-Kutta according to the flux and the force. Therefore, the key to get the static and dynamic characteristics is the calculation of the flux and the force. Finite element method (FEM) is widely used to calculate the magnetic field. However, it takes minutes even hours to solve the dynamic characteristic by FEM. Its computational efficiency limits its application in the optimization problem. It is necessary to replace the FEM by an approximate model to realize the fast calculation of static and dynamic characteristics. RBF network model is an accurate approximate model. It is suitable for the calculation of contactors' characteristics because of its fast convergence speed and strong bureau approximation ability.

The principle of RBF network is representing the objective function by the sum of a series of radial basis functions, that is, assume the objective function is [35]

$$
y=\hat{y}+\varepsilon=\sum_{i=1}^{m} \lambda_{i} \phi_{i}\left(r_{i}, c\right)+\varepsilon,
$$

where $y$ is the real value of the objectives, $\hat{y}$ is the value obtained by the RBF network, $\varepsilon$ is the error, $\lambda_{i}$ is the weight; $\varphi$ is the RBF, $r_{i}=\left\|x-x_{i}\right\|$ is the distance between the input vectors and centers, $\mathbf{x}$ is the input vectors, $x_{i}$ is the centers of the RBF, $m$ is the number of centers, and $c$ is a real constant. Equation (3) can also be expressed as

$$
\begin{aligned}
Y & =\Phi \lambda+\varepsilon, \\
\Phi & =\left[\begin{array}{ccc}
\phi_{11} & \ldots & \phi_{1 m} \\
\vdots & \ddots & \vdots \\
\phi_{n 1} & \ldots & \phi_{n m}
\end{array}\right],
\end{aligned}
$$

where $Y={ }^{T}\left[y_{1}, y_{2}, \ldots, y_{n}\right], \quad \boldsymbol{\lambda}=\left[\lambda_{1}, \lambda_{2}, \ldots, \lambda_{m}\right]^{T}$, and $\boldsymbol{\varepsilon}=$ $\left[\varepsilon_{1}, \varepsilon_{2}, \ldots, \varepsilon_{n}\right]^{T}$.

The RBF networks are built by two steps. The first step is selecting the centers of the RBF. The second is the determination of weights. The weights can be obtained by

$$
\lambda=\left(\Phi^{T} * \Phi\right)^{-1} \Phi^{T *} Y .
$$

The selection of the centers is the key to RBF network, which directly influences the accuracy of the approximate model. The most common methods of center selection are random selection, $k$-means clustering, orthogonal least square 
(OLS), and so on. In these methods, OLS is a relatively accurate method. The OLS selects the centers one by one from the sampling points by judging the contribution rate of each sampling point to the error. The RBF network built by the OLS has relatively few center points. So the RBF network is computationally efficient, and it is suitable for calculating the characteristics of contactors. Calculating the dynamic characteristics by the RBF networks needs only about $1.5 \mathrm{~s}$. The computational efficiency satisfies the need of optimization algorithms. The multiobjective optimization problem of contactor can be achieved by combining the RBF network and multiobjective optimization algorithms.

\section{NMOPSO with the Entropy Weight Ideal Point Theory}

3.1. The Multiobjective Optimization Problems. The MOPs can be described by the following equation [36]:

$$
\begin{array}{ll}
\min & \mathbf{F}(\mathbf{X})=\left(f_{1}(\mathbf{X}), f_{2}(\mathbf{X}), \ldots, f_{i}(\mathbf{X})\right), \quad i=1,2, \ldots, m, \\
\text { s.t. } & g_{j}(\mathbf{X})=0, \quad j=1,2, \ldots, s, \\
& h_{k}(\mathbf{X}) \leq 0, \quad k=1,2, \ldots, l,
\end{array}
$$

where $\mathbf{F}(\mathbf{X})$ is the objective vector, $f_{i}(\mathbf{X})$ is the $i$ th optimization objective, $m$ is the number of optimization objectives, $\mathbf{X}=\left[x_{1}, \ldots, x_{n}\right]$ is the decision vectors, and $g_{j}(\mathbf{X})$ and $h_{k}(\mathbf{X})$ are the constraints. The optimization objectives of the MOPs are all interrelated. The improvement of one objective is often accompanied by the deterioration of others. The solutions of MOPs cannot easily compare with each other, and there is not a solution that can make all the objectives reach the optimum. So the Pareto dominance is always used in the MOPs, and the Pareto optimal set is obtained in these problems instead of the only one best solution.

Definition 1. Pareto dominance: $\mathbf{X}_{a}$ and $\mathbf{X}_{b}$ are the decision vectors of MOPs, only if $\forall i=1,2, \ldots, m: f_{i}\left(\mathbf{X}_{a}\right) \leq f_{i}\left(\mathbf{X}_{b}\right)$ and $\exists i=1,2, \ldots, m: f_{i}\left(\mathbf{X}_{a}\right)<f_{i}\left(\mathbf{X}_{b}\right) ; \mathbf{X}_{a}$ dominates $\mathbf{X}_{b}$, which is defined as $\mathbf{X}_{a}<\mathbf{X}_{b}$.

Definition 2. Pareto optimal: $\mathbf{X}_{a}$ is the Pareto optimal only if $\neg \exists \mathbf{X}_{c}: \mathbf{X}_{c} \prec \mathbf{X}_{a}$.

Definition 3. Pareto front: all the objective vectors corresponding to Pareto optimal make up the Pareto front of the multiobjective problem.

3.2. NMOPSO considering the Independent Optimal Solutions. The particle swarm optimization (PSO) is proposed by Kennedy and Eberhart in 1995 [37]. They compared the optimization process with the process of birds searching for food and designed optimization strategies based on the behavior of the bird flock. The basic PSO is used for single-objective optimization. The core of PSO is the updated rules of the position and velocity of the particle. When birds are searching for food, they both follow its own experience and move to the birds who performed better. So, the velocity and position of each particle in the swarm can be updated according to the following:

$$
\begin{aligned}
v_{i j}(t+1)= & W v_{i j}(t)+c_{1} r_{1}(t)\left(P_{i j}^{*}-x_{i j}(t)\right) \\
& +c_{2} r_{2}(t)\left(P_{g j}^{*}-x_{i j}(t)\right), \\
x_{i j}(t+1)= & x_{i j}(t)+v_{i j}(t+1),
\end{aligned}
$$

where $t$ is the iterations, $v_{i j}$ is the $j$ th dimension velocity of particle $i, W$ is the inertia weight which determines whether particles have better global ability or better local ability, $c_{1}$ is the cognition weight, $c_{2}$ is the social weight. $r_{1}$ and $r_{2}$ are two random values uniformly distributed in the range of $(0,1), P_{i j}^{*}$ is the $j$ th dimension of personal best (pbest) of particle $i$, and $P_{g j}^{*}$ is the $j$ th dimension of the global best (gbest) of the swarm.

There are two main differences between MOPSO and PSO. First, the results of MOPSO are not one best solution but a Pareto optimal set. Second, the solutions in the MOPSO often cannot compare with each other; it is hard to select the $P_{i j}^{*}$ and the $P_{g j}^{*}$. In view of the above differences, the following two optimization strategies can be used in MOPSO. And the algorithm is called NMOPSO.

(1) The archiving strategy based on the niching theory. In the optimization process, an external archive is established to retain the nondominated solutions that have been obtained in the optimization process. By sorting and deleting the solutions in the external archive, the final Pareto optimal set is obtained. To maintain the diversity of the solutions in the archive, the solutions are sorted by the fitness which is obtained according to niching theory through formula (7). If the number of solutions in the archive exceeds the defined value, the last solutions is deleted:

$$
\begin{aligned}
\operatorname{Ft}(i) & =\frac{1}{\sum_{j=P_{j}} s(d(i, j))}, \\
s(d(i, j)) & = \begin{cases}1-\left(\frac{d(i, j)}{\sigma}\right)^{\alpha}, & d(i, j)<\sigma, \\
0, & \text { others, }\end{cases}
\end{aligned}
$$

where $P_{j}$ is the solutions in the archive, $\mathrm{Ft}(i)$ is the fitness of $P_{j}, s(d(i, j))$ is the sharing function of the solutions, $d(i, j)$ is the Euclidean distance between the $i$ th and $j$ th solutions, $\sigma$ is the niche radius, and $\alpha$ is a constant coefficient.

(2) The selection strategy of the pbest and the gbest. For each particle, if its new solution is dominant of its predecessors in one iteration, the new solution is set as the pbest of the particle, otherwise the new solution is set as the pbest with a probability of $50 \%$. For all the particles, the gbest is 
selected by the roulette in the external archive according to the fitness.

In analyzing the optimal solutions of each objective independently in the MOPs, the limiting case of these objectives can be obtained. These solutions are called independent optimal solution here. The independent optimal solution is representative in the optimization and can be used to evaluate the optimization effect. However, in the optimization process, NMOPSO does not care about finding the independent optimal solution for each objective but converges to the Pareto optimal set. And the particle density near the independent optimal solution is high; the independent optimal solution may be mistakenly deleted when deleting the last solutions in the archive. To solve this problem, single-objective particle swarms are added to search for the independent optimal solution of each objective. And the independent optimal solution of each objective is stored in the archive to prevent that the final Pareto optimal set does not contain the limiting case of each objective. And it also provides data support for the determination of the best compromise solution.

The NMOPSO always converges to one or more solutions prematurely because of its high convergent rate. This phenomenon is more obvious when independent optimal solutions of each objective are added. Therefore, it is necessary to add appropriate mutation in the NMOPSO to maintain the diversity of the solution. Here, each dimension of the particles is mutated with a probability $\mathrm{Cb}$ before each circle, where $\mathrm{Cb}$ is calculated by the following:

$$
\mathrm{Cb}=\mathrm{Cb}_{0}\left(1-\frac{t}{N}\right)^{e}
$$

where $\mathrm{Cb}$ is the mutation probability, $\mathrm{Cb}_{0}$ is the initial mutation probability, $t$ is the iterations, $N$ is the maximum iterations, and $e$ is a constant coefficient. $\mathrm{Cb}$ initially declines fast and then tends to be flat. That ensures the PSO has strong global search ability at the beginning of the optimization process, to avoid falling into the local optimal solution. At the same time, PSO can quickly converge to the Pareto optimal set at the end of the optimization process.

According to the above strategy, the Pareto optimal set can be obtained by 8 steps.

Step 1. Initialize the multiobjective particle swarm, the single-objective particle swarms, and the external archive.

Step 2. Search the independent optimal solutions of each objective by the single-objective particle swarms.

Step 3. Calculate the value of each particle in the multiobjective particle swarm according to $\mathbf{F}(\mathbf{X})$. Keep the nondominated solutions and the independent optimal solutions in the external archive.

Step 4. Delete the dominated solutions and the repeated solutions in the archive. Calculate the fitness of the solutions in the archive. Sort the solutions by the fitness, and if the number of solutions in the archive exceeds the defined value, delete the last solutions.

Step 5. Select the gbest by the roulette in the external archive according to the fitness.
Step 6. For each particle in the present iterations, judge if it is dominated by the previous solution and select the pbest.

Step 7. Update the velocity and position of each particle according formula (6). Mutate the particle according to formula (8).

Step 8. If the number of iterations is equal to $N$, stop the algorithm or else return to Step 3.

In order to verify the performance of the NMOPSO proposed in this paper, 3 standard test functions ZDT1, ZDT2, and ZDT3 are used [38]. The Pareto front of ZDT1 is convex, the Pareto front of ZDT2 is concave, and the Pareto front of ZDT3 is discontinuous. According to formula (9), the distance between the solutions in the archive and the true Pareto front can be calculated. That is called the generational distance [39].

$$
\mathrm{GD}=\frac{\sqrt{\sum_{i=1}^{n} \mathrm{dist}_{i}^{2}}}{n},
$$

where $n$ is the number of solutions in the archive and dist ${ }_{i}$ is the Euclidean distance between the objective vector of $i$ th nondominated solution and the nearest member of true Pareto optimal set. GD $=0$ means all the nondominated solutions are on the Pareto front.

The NMOPSO is compared with two classical algorithmsNSGA-II and MOPSO. Moreover, the NMOPSO is also compared with two typical up-to-date algorithms-AGMOPSO and CDMOPSO [14]. Here, the number of particles is set to 50 and the number of nondominated solutions in the archive is set to 100 . The maximum iterations are set to 100 . The results of each algorithm are obtained from 20 times independent runs. Figure 1 shows the typical figure of each algorithm and Table 1 shows the comparison results.

Figures 1(a)-1(c) show the comparison of NMOPSO with classical algorithms, and Figures 1(d)-1(f) show the comparison of NMOPSO with up-to-date algorithms. In Figure 1, all five algorithms can converge to the Pareto optimal set and have good diversity. However, from the data in Table 1, NMOPSO works significantly better than others when ZDT3 was optimized and can get the same even better results compared with up-to-date algorithms when ZDT1 and ZDT2 are optimized. At the same time, the NMOPSO proposed in this paper can better get the limiting case of each objective because of the searching and archiving strategy of the independent optimal solutions. Especially for ZDT3, NMOPSO can get the limiting case of objective more reliably. NMOPSO in this paper can get the limiting case of each objective and is helpful to determine the best compromise solution.

\subsection{Selection of Compromise Solution Based on the Entropy} Weight Ideal Point Theory. The result obtained by NMOPSO is a Pareto optimal set; however, in an engineering application, a decision is needed to select a compromise solution in the set. Here, an entropy weight ideal point theory is proposed to select the best compromise solution.

Ideal point is an important definition in the MOP. For multiobjective optimization problems, the Pareto optimal set is $\mathbf{P}$, if the $f_{i}^{0}$ satisfy $(10)$. The $F^{0}=\left(f_{1}^{0}, f_{2}^{0}, \ldots, f_{m}^{0}\right)$ is 


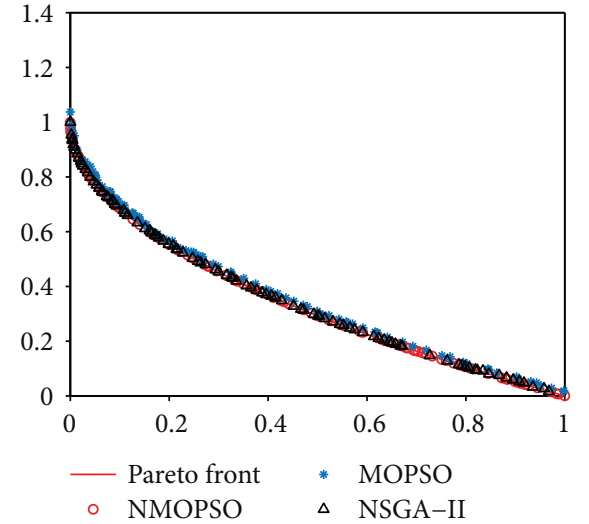

(a) ZDT1

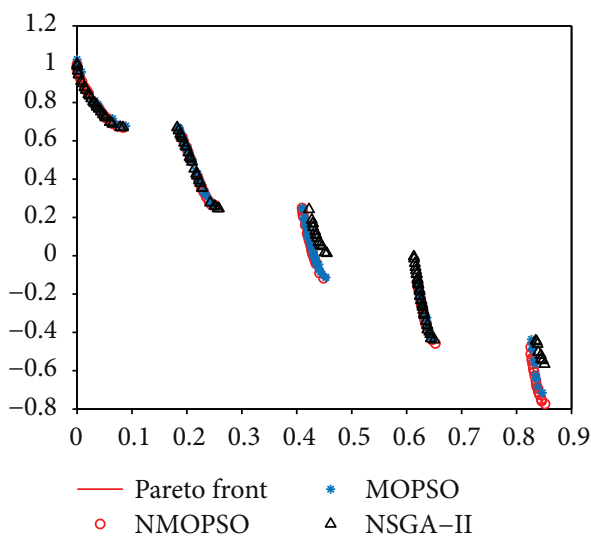

(c) ZDT3

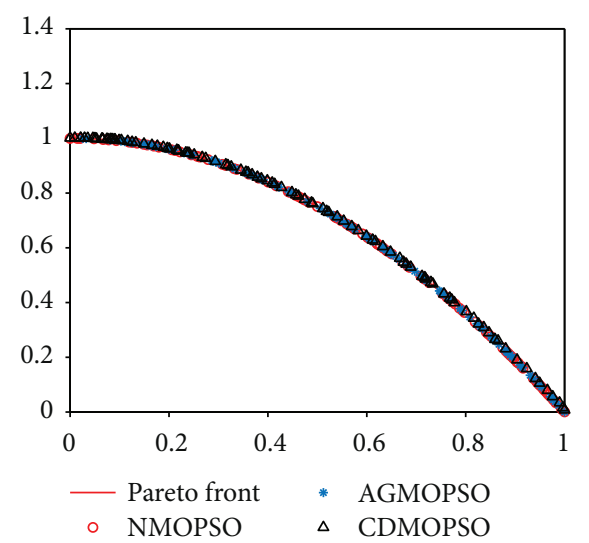

(e) ZDT2

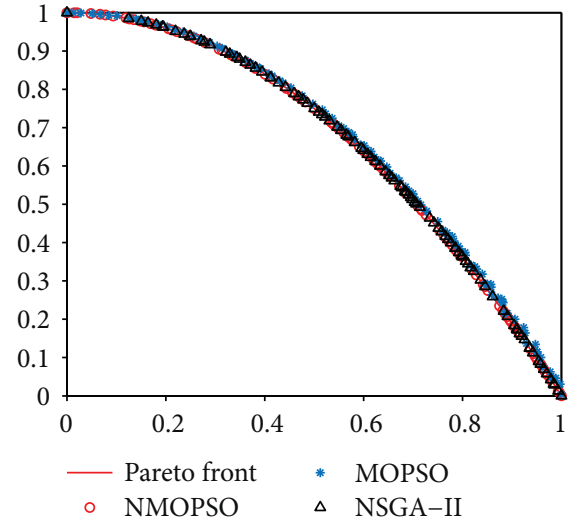

(b) ZDT2

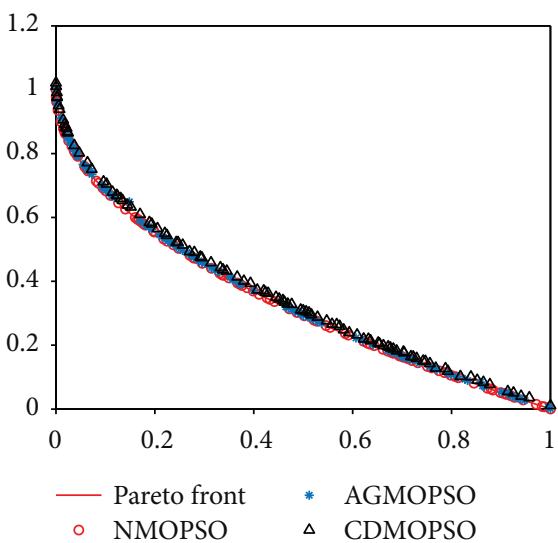

(d) ZDT1

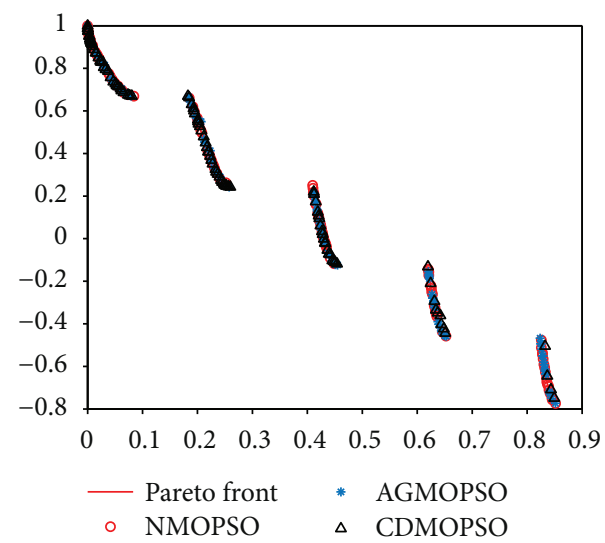

(f) ZDT3

FIGURE 1: The performance of five algorithms.

called the ideal point of this MOP, which is corresponding to the independent optimal solutions of each objective.

$$
f_{i}^{0} \leq \min _{x \in \mathrm{P}} f_{i}(\mathbf{X}), \quad \forall i=1,2, \ldots, m .
$$

Due to the mutual restraint relationship among the objectives in the MOP, the ideal point cannot be obtained generally. The ideal point method is to find $\mathbf{X}$ on the solution set which makes the distance between the $\mathbf{F}(\mathbf{X})$ and $F^{0}$ to be minimum by solving the following:

$$
\min _{x \in P} U(\mathbf{X})=\left\|F(\mathbf{X})-F^{0}\right\|=\left(\sum_{i=1}^{m} w_{i}\left(f_{i}(\mathbf{X})-f_{i}^{0}\right)^{2}\right)^{1 / 2},
$$

where $w_{i}$ is the inertia weight. Because the unit and the magnitude of each objective are different, the $w$ in (11) needs to 
TABLE 1: The performance of five algorithms.

\begin{tabular}{|c|c|c|c|c|c|c|c|}
\hline & & & NMOPSO & MOPSO & NSGA-II & AGMOPSO & CDMOPSO \\
\hline \multirow{6}{*}{ ZDT1 } & \multirow{2}{*}{ GD } & Mean & $1.49 e-4$ & $2.02 e-3$ & $1.01 e-3$ & $6.23 e-4$ & $7.80 e-4$ \\
\hline & & Variance & $1.82 e-9$ & $1.12 e-5$ & $7.70 e-7$ & $3.17 e-6$ & $2.02 e-7$ \\
\hline & \multirow{2}{*}{ Obj1 } & Best & 0 & 0 & 0 & 0 & 0 \\
\hline & & Worst & 0 & 0.009 & 0 & 0 & 0 \\
\hline & \multirow{2}{*}{ Obj2 } & Best & 0 & 0.002 & 0 & 0 & 0 \\
\hline & & Worst & 0 & 0.178 & 0.329 & 0.031 & 0.050 \\
\hline \multirow{6}{*}{ ZDT2 } & \multirow{2}{*}{ GD } & Mean & $9.14 e-5$ & $5.28 e-4$ & $9.94 e-4$ & $9.61 e-5$ & $1.23 e-4$ \\
\hline & & Variance & $2.58 e-8$ & $2.91 e-8$ & $4.76 e-7$ & $2.25 e-9$ & $6.75 e-9$ \\
\hline & \multirow{2}{*}{ Obj1 } & Best & 0 & 0 & 0 & 0 & 0 \\
\hline & & Worst & 0 & 0 & 0 & 0.011 & 0 \\
\hline & \multirow{2}{*}{ Obj2 } & Best & 0 & 0 & 0 & 0 & 0 \\
\hline & & Worst & 0.027 & 0.040 & 0.243 & 0.037 & 0.004 \\
\hline \multirow{6}{*}{ ZDT3 } & \multirow{2}{*}{ GD } & Mean & $1.53 e-4$ & $4.65 e-4$ & $2.70 e-3$ & $3.31 e-3$ & $1.22 e-3$ \\
\hline & & Variance & $4.32 e-9$ & $3.05 e-8$ & $6.61 e-6$ & $3.17 e-5$ & $9.75 e-6$ \\
\hline & \multirow{2}{*}{ Obj1 } & Best & 0 & 0 & 0 & 0 & 0 \\
\hline & & Worst & 0 & 0.002 & 0 & 0 & 0 \\
\hline & \multirow[t]{2}{*}{ Obj2 } & Best & -0.773 & -0.744 & -0.773 & -0.773 & -0.773 \\
\hline & & Worst & -0.773 & -0.506 & -0.355 & -0.592 & -0.563 \\
\hline
\end{tabular}

be determined according to the actual situation of each objective. In order to avoid the influence of subjective experience, an entropy weight method is used to determine the weight here. And the relative rate of change $\left(f_{i}(\mathbf{X})-f_{i}^{0}\right) / \max _{x \in P} \mid f_{i}$ $(\mathbf{X}) \mid$ is used here to replace the $\left(f_{i}(\mathbf{X})-f_{i}^{0}\right)$ so that can eliminate the impact of the unit and characterize the degree of change of each objective more accurately.

The entropy weight method determines the weight according to the amount of information carried by each objective. In this method, the higher amount of information the objective contains, the bigger the weight of the objective is. In order to obtain the entropy weight of each objective in the Pareto solution set, each objective should be standardized according to the following:

$$
\begin{aligned}
\mathrm{pb}_{i j} & =\frac{\mathrm{pk}_{i j}-\min \left(\mathrm{pk}_{i}\right)}{\max \left(\mathrm{pk}_{i}\right)-\min \left(\mathrm{pk}_{i}\right)}, \\
E_{i} & =\frac{\sum_{j=1}^{n} \mathrm{pe}_{i j} \ln \mathrm{pe}_{i j}}{-\ln n}, \\
w_{i} & =\frac{1-E_{i}}{\sum_{i=1}^{m}\left(1-E_{i}\right)},
\end{aligned}
$$

where $\mathrm{PK}=\left(\mathrm{pk}_{i j}\right)$ is a nondominated solution set containing $n$ solutions and $m$ objectives, $i=1,2, \ldots, n$ and $j=1$, $2, \ldots, m$, respectively. $\mathrm{pb}_{i j}$ is the standardized data. $E_{i}$ is the entropy of the $i$ th objective. $\mathrm{pe}_{i j}=\mathrm{pb}_{i j} / \sum_{j=1}^{n} \mathrm{pb}_{i j}$ is the probability of each value of the $i$ th objective if $\mathrm{pe}_{i j}=1 / n$, $j=1,2, \ldots, n$. That the probability of each value is the same means this objective contains no information and the weight is set to 0 .

\section{The Optimization Results of Contactor}

The structure of the typical direct-acting contactor is clear and its working principle is simple. So here, a typical directacting contactor is selected to verify the effect of NMOPSO proposed in this paper. The structure of the contactor is shown in Figure 2. Select the retention force of the contactor, making time, coil power, and the mass of the armature as the optimization objectives to represent the static and dynamic characteristics of the contactor, power consumption, and other needs. Here, the retention force of the contactor is expected to be bigger, while other objectives are expected to be smaller. The main parameters that affect the objectives are the size parameters $X_{1}, R_{1}, R_{2}, R_{3}$, and the coil resistance $\left(R_{\Omega}\right)$. To ensure there is no interference between the components of the contactor, the initial values and constraints of each parameter are given in Table 2.

In the above four objectives, the coil power and the mass of armature can be directly calculated according to the coil resistance and the size parameters. However, the retention force of the contactor and the making time need to be obtained by establishing the approximate model of contactors' static and dynamic characteristics. The dynamic characteristics of the contactor are obtained by iterative solution of the static characteristic. To ensure the effect of the approximate model, only the accuracy of solving the dynamic characteristic of the contactor needs to be verified. Here, get the coil current by the oscilloscope monitors and the armature displacement by the laser displacement sensor. Figure 3 shows the comparison of the RBF network approximate model results, the FEM results, and the actual data.

In Figure 3, the three curves of results basically coincide. The making time of this contactor is $23 \mathrm{~ms}$ in practice, the 


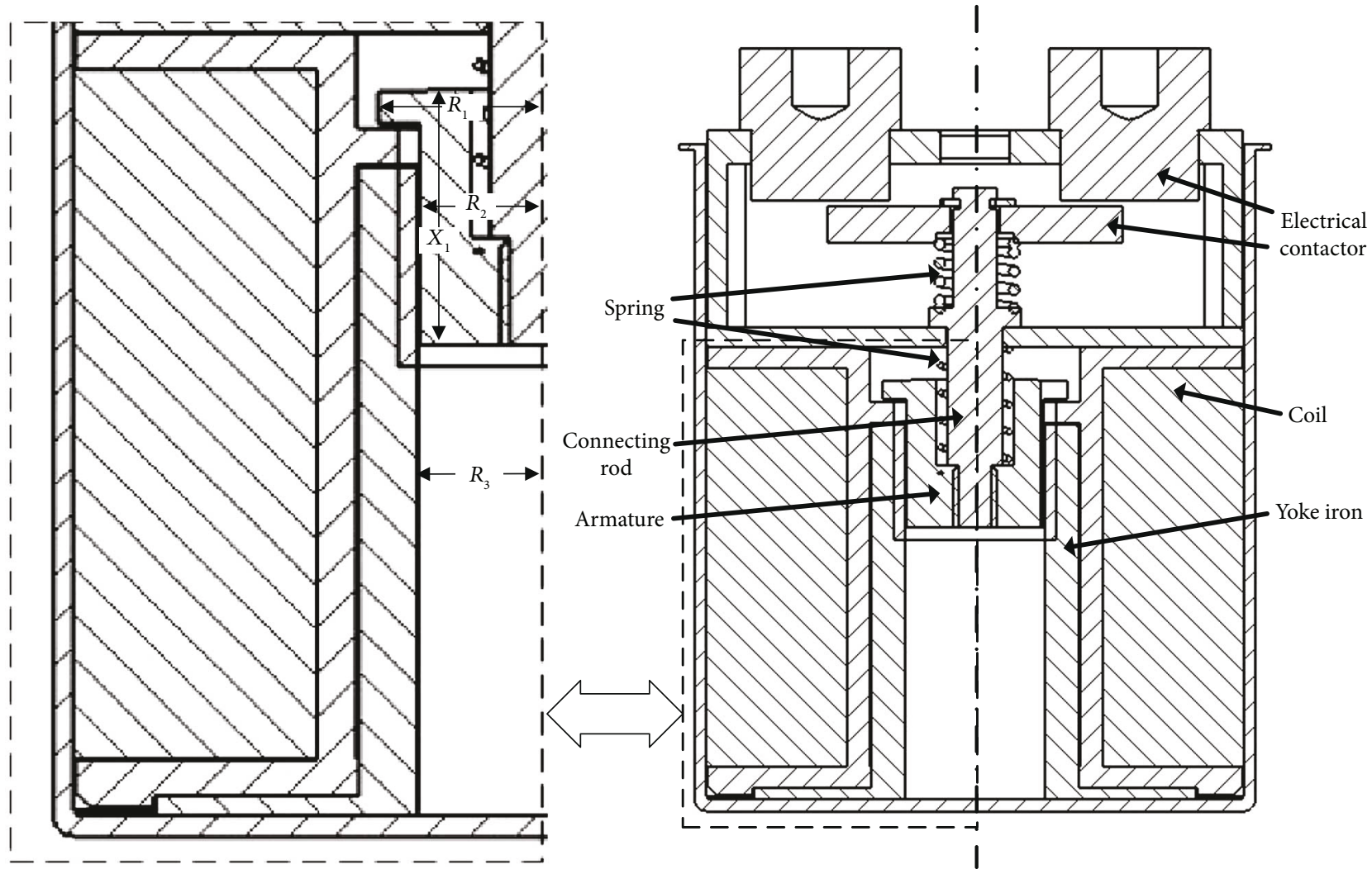

Figure 2: Structure of the typical direct-acting contactor.

TABLE 2: The initial values of each parameter.

\begin{tabular}{lcc}
\hline Parameters & Initial values & Constraints \\
\hline$R_{1}$ & $8.00 \mathrm{~mm}$ & $(7,9) \mathrm{mm}$ \\
$R_{2}$ & $6.00 \mathrm{~mm}$ & $(5.5,6.5) \mathrm{mm}$ \\
$R_{3}$ & $6.00 \mathrm{~mm}$ & $(6,7) \mathrm{mm}$ \\
$X_{1}$ & $12.80 \mathrm{~mm}$ & $(11.8,13.8) \mathrm{mm}$ \\
$R_{\Omega}$ & $40.00 \Omega$ & $(36,44) \Omega$ \\
\hline
\end{tabular}

making time is $22.6 \mathrm{~ms}$ by the FEM, and the making time is $22 \mathrm{~ms}$ by the approximate model. The error is within $5 \%$. The approximate model needs $1.5 \mathrm{~s}$ to calculate the dynamic characteristics; however, the FEM requires $15 \mathrm{~min}$. The approximate model greatly improves the computational efficiency. It can be seen that the accuracy and computational efficiency of the approximate model meet the requirements of NMOPSO and can be used for the MOP of contactors.

To verify the performance of the NMOPSO used in the field of contactor, here the retention force of the contactor and the making time are optimized, and the result is compared with the MOPSO. Here, the number of particles is set to 50 , and the number of particles in the archive is set to 100 in three algorithms. The maximum iterations are set to 100. Figure 4 shows the Pareto front of the force and making time. Both of the two algorithms can find the Pareto front of the MOP. However, due to the mutation and the retention of independent optimal solution, the results obtained by the NMOPSO are more closed to the limiting case of each objective, and some solutions obtained by the NMOPSO obviously outperform those by the MOPSO. The performance of the NMOPSO is better and suits the optimization of the contactor.

Using the NMOPSO, optimize the above four contactor's objectives which are the retention force of the contactor, making time, coil power, and the mass of the armature. The entropy weight of each objective is calculated by formula (12), and the independent optimal solution of each objective is recorded in Table 3. It can be seen that the objectives of the contactor have a great optimized space according to Table 3.

The result of each objective after the optimization is shown in Table 4. In these objectives, the optimization of the retention force of the contactor and the coil power is more obvious. The force is increased by $32.3 \mathrm{~N}$, the coil power is decreased by $1.2 \mathrm{~W}$, and the making time and the mass are not obviously improved but still maintain the original level. At this point, if a parameter is changed to improved one objective, the other objectives will be worse. For example, reducing the coil resistance to $40 \Omega$ and keeping the other parameters constant will reduce the making time to $21.3 \mathrm{~ms}$ and increase the rate of optimization from $1.8 \%$ to $3.2 \%$. However, that will make the coil power increase to $19.6 \mathrm{~W}$ and the rate of optimization from $6.1 \%$ to $0 \%$. It can be seen that the compromise solution optimized by the entropy weight ideal point method is relatively better. 


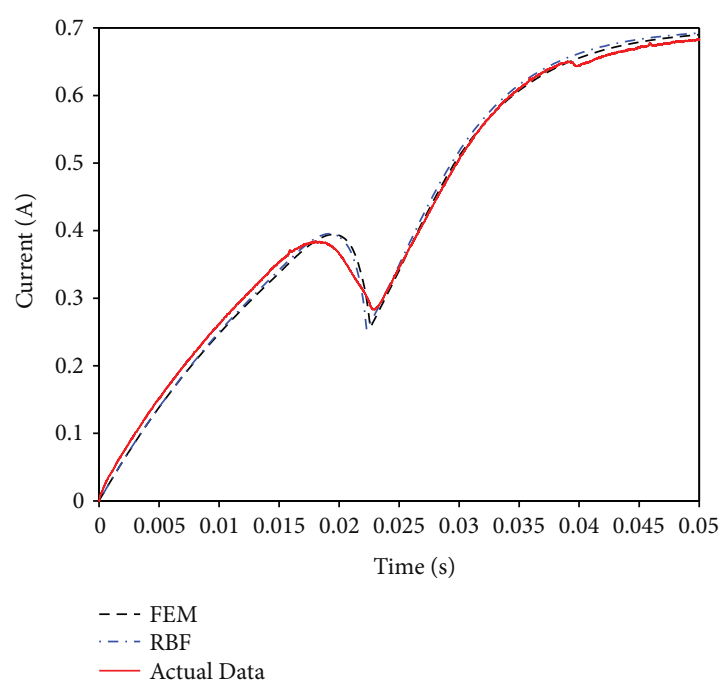

(a) The coil current of contactor

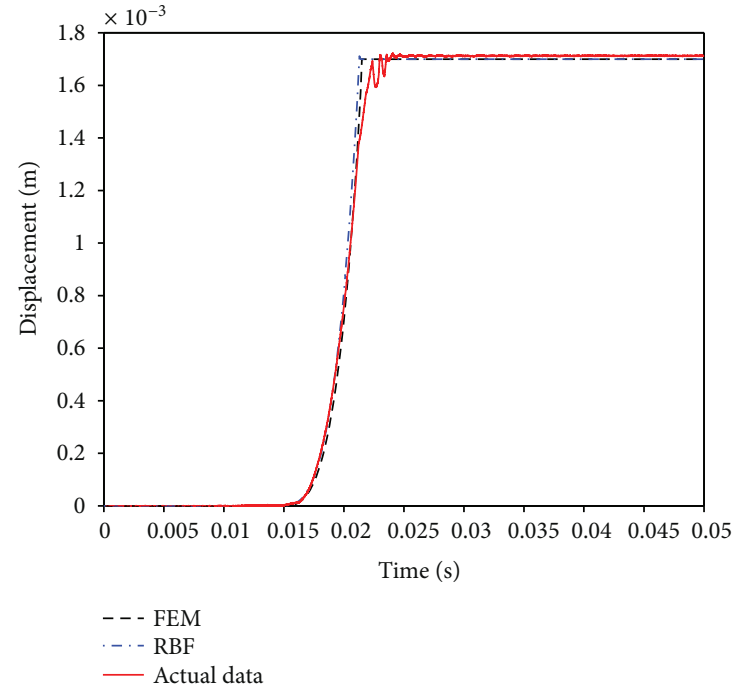

(b) The displacement of armature

FIGURE 3: The comparison of the accuracy of the approximate model.
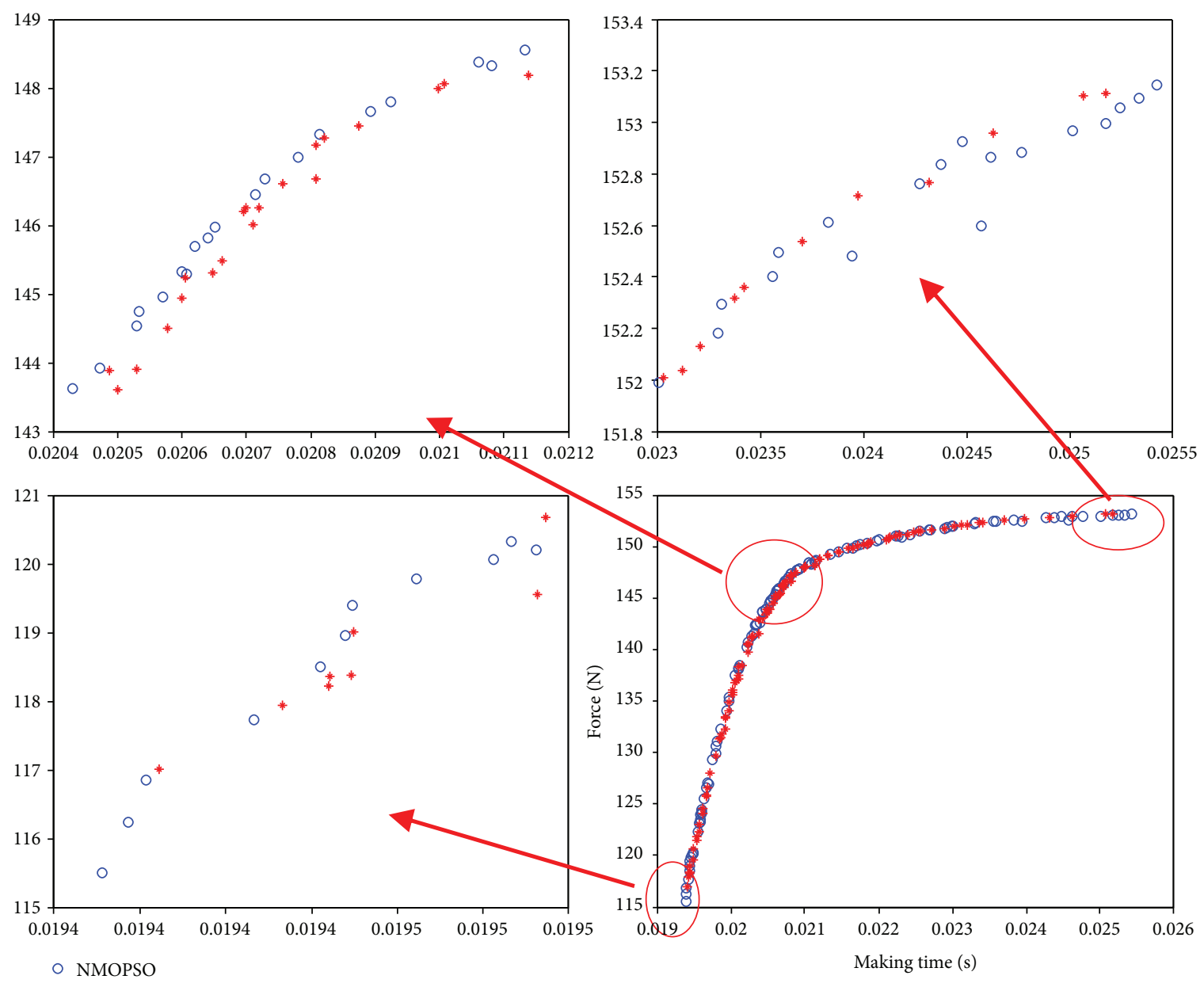

* MOPSO

FIgure 4: The Pareto front of the force and making time. 
TABLE 3: The optimal and initial values of each objective.

\begin{tabular}{lcccc}
\hline Objectives & Initial values & Optimal & Rate & Weight \\
\hline Making time & $22 \mathrm{~ms}$ & $19.4 \mathrm{~ms}$ & $-11.8 \%$ & 0.21 \\
Retention force & $107.7 \mathrm{~N}$ & $153.1 \mathrm{~N}$ & $+42.2 \%$ & 0.1 \\
Coil power & $19.6 \mathrm{~W}$ & $17.8 \mathrm{~W}$ & $-9.2 \%$ & 0.55 \\
Mass & $0.0204 \mathrm{~kg}$ & $0.0177 \mathrm{~kg}$ & $-13.2 \%$ & 0.14 \\
\hline
\end{tabular}

TABLE 4: The final optimal results.

\begin{tabular}{lccc}
\hline Objectives & Initial values & Results & Rate \\
\hline Making time & $22 \mathrm{~ms}$ & $21.6 \mathrm{~ms}$ & $-1.8 \%$ \\
Retention force & $107.7 \mathrm{~N}$ & $140.0 \mathrm{~N}$ & $+30.0 \%$ \\
Coil power & $19.6 \mathrm{~W}$ & $18.4 \mathrm{~W}$ & $-6.1 \%$ \\
Mass & $0.0204 \mathrm{~kg}$ & $0.0204 \mathrm{~kg}$ & $0 \%$ \\
\hline
\end{tabular}

\section{Conclusion}

For solving the MOP of the contactors, a NMOPSO which considers the independent optimal solutions and mutation is proposed in this paper. The NMOPSO realizes selecting and sorting of the nondominated solutions by archiving strategy which is based on the niching technique. And an entropy weight ideal point idea is also contained in this method to get the best compromise solution.

Taking a typical high-power DC contactor as an example, an approximate model of the static and dynamic characteristics of the contactor is established based on the RBF network so that the solving efficiency of the objectives of the static and dynamic characteristics satisfies the requirements of the MOP. Then, the objectives such as the retention force of the contactor, making time, coil power, and the mass of the armature are optimized by the NMOPSO proposed in this paper, and the best compromise solution is determined. After the optimization, the retention force of the contactor has increased $30 \%$, and the coil power has reduced $6 \%$. The other two objectives maintain the original level. In conclusion, the method proposed in this paper is good at the optimization of the contactors and can get good optimization result. The multiobjective optimization algorithm also suits the similar field with good promotion.

\section{Data Availability}

The data used to support the findings of this study are available from the corresponding author upon request.

\section{Conflicts of Interest}

The authors declare that they have no conflicts of interest.

\section{Acknowledgments}

This research work was supported by the National Natural Science Foundation of China under Grant no. 51707044.

\section{References}

[1] W. Qiya, Z. Guofu, C. Xianke, and C. Zhijun, "Key adjusted parameters and optimum method of electromagnetic relay based on orthogonal experimental design," Transactions of China Electrotechnical Society, vol. 24, no. 10, pp. 53-58, 2009.

[2] L. Huimin, Y. Haidan, T. Yujie, Z. Chunqing, Z. Wen, and Z. Han, "Virtual prototyping and parameter optimization of differential electromagnetic relays with double permanent magnets," Proceedings of the CSEE, vol. 36, no. 1, pp. 258-267, 2016.

[3] Z. Guofu, W. Qiya, C. Xianke, and Z.-j. Chen, "Fast algorithm of dynamic characteristics of electromagnetic relay and its application on optimization," Proceedings of the CSEE, vol. 30, no. 12, pp. 106-110, 2010.

[4] W. Yang, P. Liu, Y. Wang, R. Li, and G. Zhai, "Research on dynamic characteristics optimization via analytical modeling for typical rotational electromagnetic system," IEEE Transactions on Magnetics, vol. 50, no. 11, pp. 1-4, 2014.

[5] Y. Li, S. Xiao, M. Rotaru, and J. K. Sykulski, "A dual kriging approach with improved points selection algorithm for memory efficient surrogate optimization in electromagnetics," IEEE Transactions on Magnetics, vol. 52, no. 3, pp. 1-4, 2016.

[6] C. M. Fonseca and P. J. Fleming, "Genetic algorithms for multipobjective optimization: formulation discussion and generalization," Proceedings of International Conference On Genetic Algorithms, no. 5, pp. 416-423, 1993.

[7] J. Horn, N. Nafpliotis, and D. E. Goldberg, "A niched Pareto genetic algorithm for multiobjective optimization," in Proceedings of the First IEEE Conference on Evolutionary Computation. IEEE World Congress on Computational Intelligence, vol. 1, pp. 82-87, Orlando, FL, USA, June 1994.

[8] N. Srinivas and K. Deb, "Muiltiobjective optimization using nondominated sorting in genetic algorithms," Evolutionary Computation, vol. 2, no. 3, pp. 221-248, 1994.

[9] K. Deb, A. Pratap, S. Agarwal, and T. Meyarivan, "A fast and elitist multiobjective genetic algorithm: NSGA-II," IEEE Transactions on Evolutionary Computation, vol. 6, no. 2, pp. 182-197, 2002.

[10] Q. Zhang and H. Li, "MOEA/D: a multiobjective evolutionary algorithm based on decomposition," IEEE Transactions on Evolutionary Computation, vol. 11, no. 6, pp. 712-731, 2007.

[11] H. L. Liu, F. Gu, and Q. Zhang, "Decomposition of a multiobjective optimization problem into a number of simple multiobjective subproblems," IEEE Transactions on Evolutionary Computation, vol. 18, no. 3, pp. 450-455, 2014.

[12] B. Y. Qu, P. N. Suganthan, and S. Das, "A distance-based locally informed particle swarm model for multimodal optimization," IEEE Transactions on Evolutionary Computation, vol. 17, no. 3, pp. 387-402, 2013.

[13] B. Xue, M. Zhang, and W. N. Browne, "Particle swarm optimization for feature selection in classification: a multi-objective approach," IEEE Transactions on Cybernetics, vol. 43, no. 6 , pp. 1656-1671, 2013.

[14] H. Han, W. Lu, L. Zhang, and J. Qiao, "Adaptive gradient multiobjective particle swarm optimization," IEEE Transactions on Cybernetics, pp. 1-13, 2017.

[15] K. E. Parsopoulos and M. N. Vrahatis, "Particle swarm optimization method in multiobjective problems," in SAC '02 Proceedings of the 2002 ACM symposium on Applied computing, pp. 603-607, Madrid, Spain, March 2002. 
[16] C. A. C. Coello, G. T. Pulido, and M. S. Lechuga, "Handling multiple objectives with particle swarm optimization," IEEE Transactions on Evolutionary Computation, vol. 8, no. 3, pp. 256-279, 2004.

[17] R. Brits, A. P. Engelbrecht, and F. V. D. Bergh, "A niching particle swarm optimizer," in Conference on Simulated Evolution and Learning, pp. 692-696, Singapore, 2002.

[18] M. N. Al, A. Petrovski, and J. Mccall, “ $D^{2} M O P S O$ : MOPSO based on decomposition and dominance with archiving using crowding distance in objective and solution spaces," Evolutionary Computation, vol. 22, no. 1, pp. 47-77, 2014.

[19] G. G. Yen and W. F. Leong, "Dynamic multiple swarms in multiobjective particle swarm optimization," IEEE Transactions on Systems, Man, and Cybernetics - Part A: Systems and Humans, vol. 39, no. 4, pp. 890-911, 2009.

[20] Q. Lin, J. Li, Z. Du, J. Chen, and Z. Ming, "A novel multiobjective particle swarm optimization with multiple search strategies," European Journal of Operational Research, vol. 247, no. 3, pp. 732-744, 2015.

[21] Q. Zhu, Q. Lin, W. Chen et al., "An external archive-guided multiobjective particle swarm optimization algorithm," IEEE Transactions on Cybernetics, vol. 47, no. 9, pp. 2794-2808, 2017.

[22] W. Hu and G. G. Yen, "Adaptive multiobjective particle swarm optimization based on parallel cell coordinate system," IEEE Transactions on Evolutionary Computation, vol. 19, no. 1, pp. 1-18, 2015.

[23] N. Al Moubayed, A. Petrovski, and J. McCall, " $D^{2} M O P S O$ : multi-objective particle swarm optimizer based on decomposition and dominance," in Evolutionary Computation in Combinatorial Optimization. EvoCOP 2012, J. K. Hao and M. Middendorf, Eds., vol. 7245 of Lecture Notes in Computer Science, pp. 75-86, Springer, Berlin, Heidelberg, 2012.

[24] A. Britto and A. Pozo, "Using reference points to update the archive of MOPSO algorithms in many-objective optimization," Neurocomputing, vol. 127, no. 3, pp. 78-87, 2014.

[25] M. Campos, R. A. Krohling, and I. Enriquez, "Bare bones particle swarm optimization with scale matrix adaptation," IEEE Transactions on Cybernetics, vol. 44, no. 9, pp. 15671578, 2014.

[26] Y. Li, Z. H. Zhan, S. Lin, J. Zhang, and X. Luo, "Competitive and cooperative particle swarm optimization with information sharing mechanism for global optimization problems," Information Sciences, vol. 293, no. 3, pp. 370-382, 2015.

[27] Q. Qin, S. Cheng, Q. Zhang, L. Li, and Y. Shi, "Particle swarm optimization with interswarm interactive learning strategy," IEEE Transactions on Cybernetics, vol. 46, no. 10, pp. 22382251, 2016.

[28] J. Li, J. Q. Zhang, C. J. Jiang, and M. C. Zhou, "Composite particle swarm optimizer with historical memory for function optimization," IEEE Transactions on Cybernetics, vol. 45, no. 10, pp. 2350-2363, 2015.

[29] W. N. Chen, J. Zhang, Y. Lin et al., "Particle swarm optimization with an aging leader and challengers," IEEE Transactions on Evolutionary Computation, vol. 17, no. 2, pp. 241-258, 2013.

[30] S. Helwig, J. Branke, and S. Mostaghim, "Experimental analysis of bound handling techniques in particle swarm optimization," IEEE Transactions on Evolutionary Computation, vol. 17, no. 2, pp. 259-271, 2013.
[31] Y. Zhang, D. W. Gong, and J. H. Zhang, "Robot path planning in uncertain environment using multi-objective particle swarm optimization," Neurocomputing, vol. 103, no. 2, pp. 172-185, 2013.

[32] Y. J. Zheng, H. F. Ling, J. Y. Xue, and S. Y. Chen, "Population classification in fire evacuation: a multiobjective particle swarm optimization approach," IEEE Transactions on Evolutionary Computation, vol. 18, no. 1, pp. 70-81, 2014.

[33] F. Sheikholeslami and N. J. Navimipour, "Service allocation in the cloud environments using multi-objective particle swarm optimization algorithm based on crowding distance," Swarm and Evolutionary Computation, vol. 35, pp. 53-64, 2017.

[34] L. Wenying, X. Chang, W. Jing, W. Jiaming, and W. Weizhou, "Optimization of transmission network maintenance scheduling based on niche multi-objective particle swarm algorithm," Proceedings of the CSEE, vol. 33, no. 4, pp. 141-148, 2013.

[35] S. Chen, C. F. N. Cowan, and P. M. Grant, "Orthogonal least squares learning algorithm for radial basis function networks," IEEE Transactions on Neural Network, vol. 2, no. 2, pp. 302309, 1991.

[36] D. A. Van Veldhuizen and G. B. Lamont, "Multiobjective evolutionary algorithms: analyzing the state-of-the-art," Evolutionary Computation, vol. 8, no. 2, pp. 125-147, 2014.

[37] J. Kennedy and R. Eberhart, "Particle swarm optimization," in Proceedings of ICNN'95 - International Conference on Neural Networks, pp. 1942-1948, Perth, WA, Australia, December 1995.

[38] E. Zitzler, K. Deb, and L. Thiele, "Comparison of multiobjective evolutionary algorithms: empirical results," Evolutionary Computation, vol. 8, no. 2, pp. 173-195, 2014.

[39] D. A. V. Veldhuizen and G. B. Lamont, "Evolutionary computation and convergence to a Pareto front," Stanford University California, 1998. 


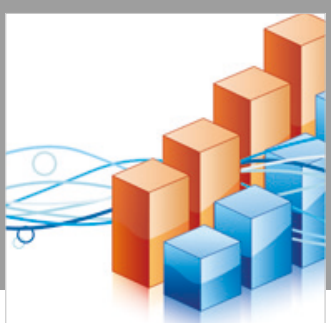

Advances in

Operations Research

\section{-n-m}
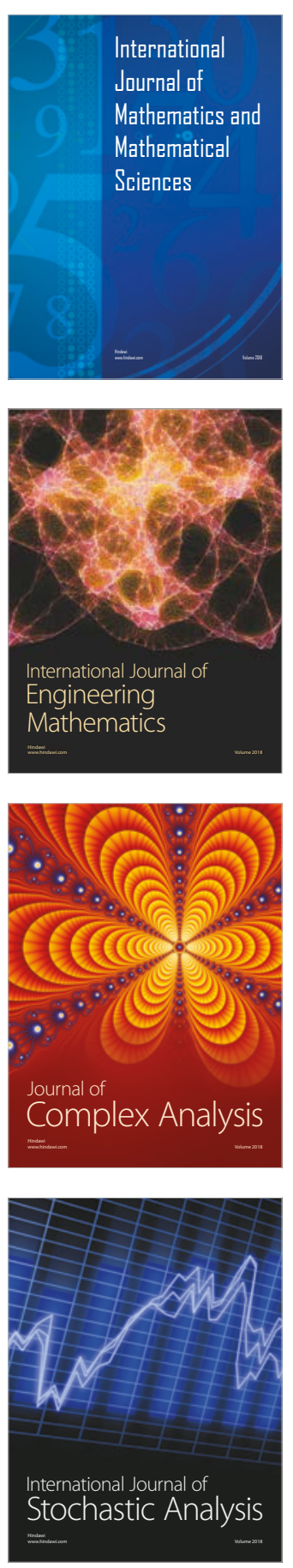
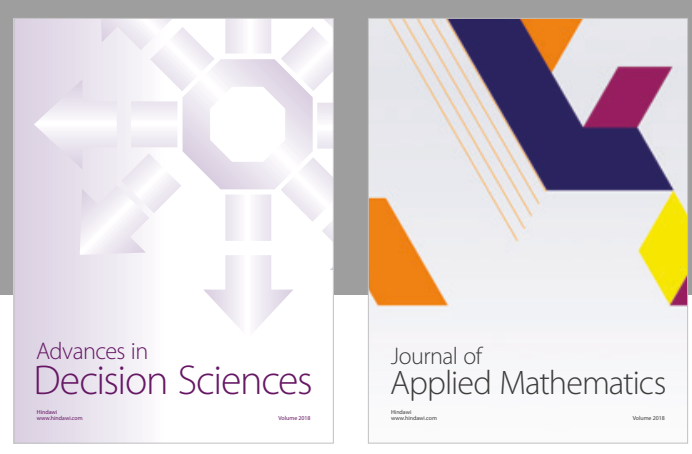

Journal of

Applied Mathematics
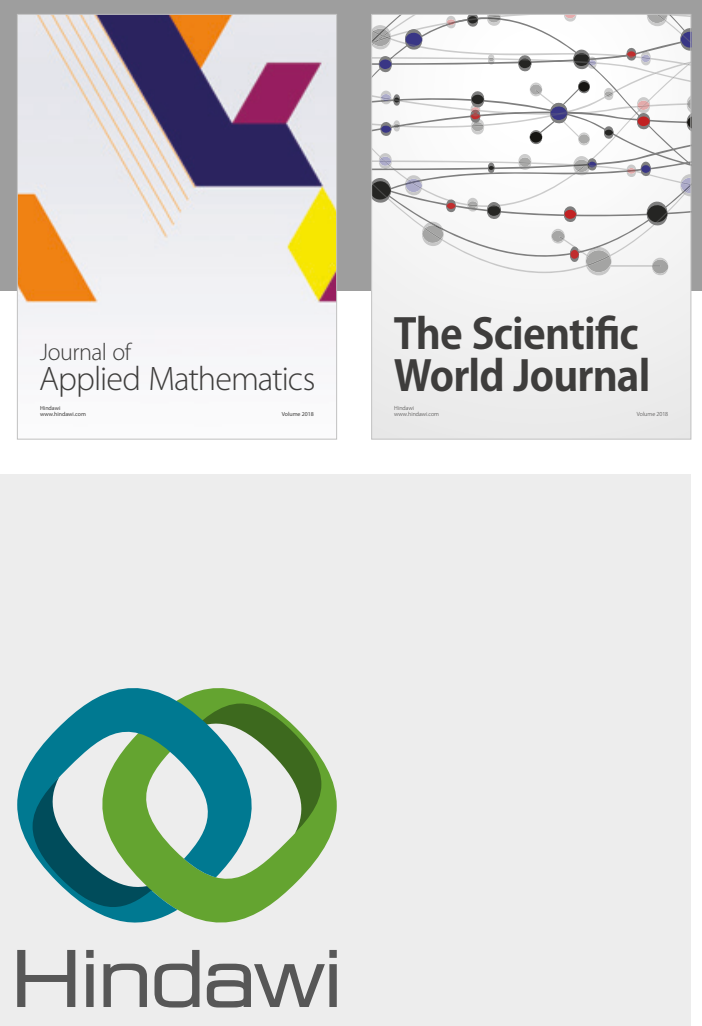

Submit your manuscripts at

www.hindawi.com

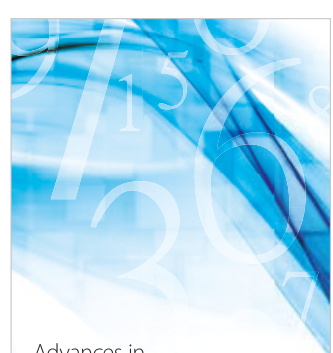

Advances in
Numerical Analysis
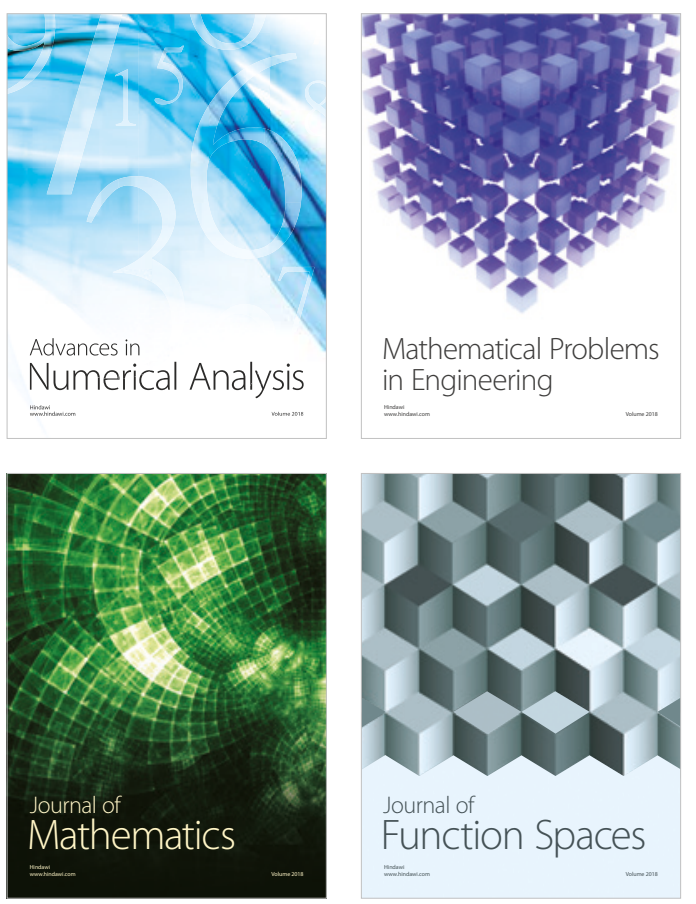

Mathematical Problems in Engineering

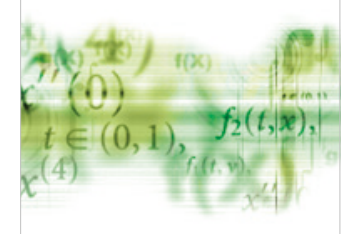

International Journal of

Differential Equations

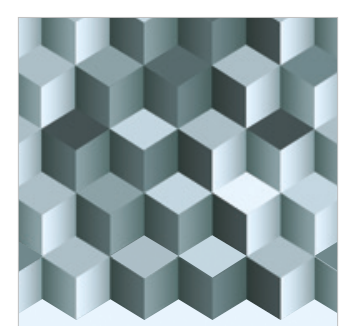

Journal of

Function Spaces
The Scientific

World Journal

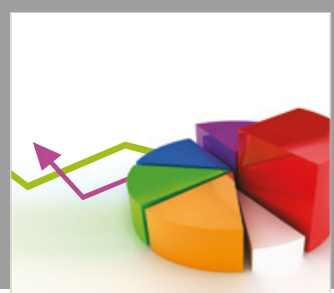

Journal of

Probability and Statistics
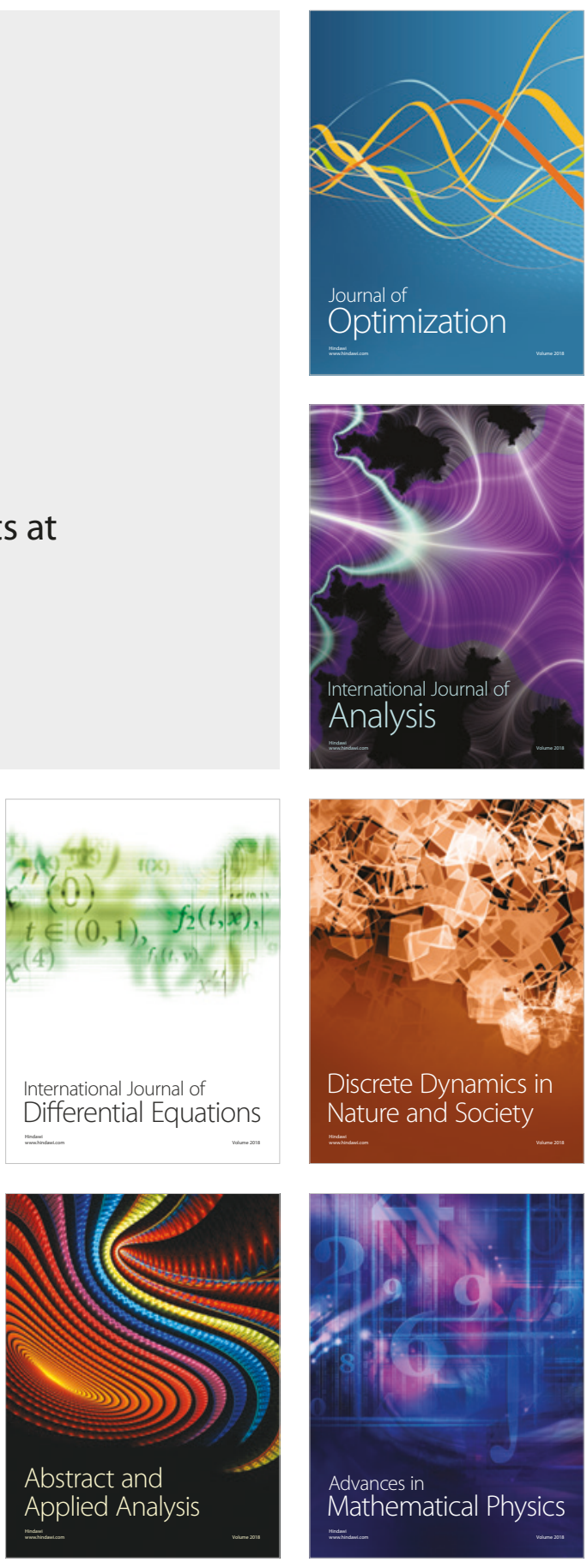